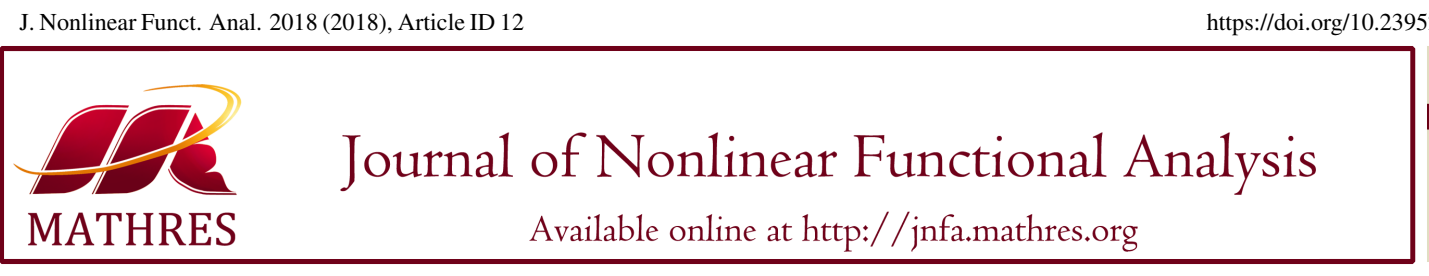

https://doi.org/10.23952/jnfa.2018.12

\title{
ON A COUPLED SYSTEM OF HILFER AND HILFER-HADAMARD FRACTIONAL DIFFERENTIAL EQUATIONS IN BANACH SPACES
}

\author{
SAÏD ABBAS ${ }^{1}$, MOUFFAK BENCHOHRA ${ }^{2, *}$, JAMAL E. LAZREG ${ }^{2}$, JUAN J. NIETO ${ }^{3}$ \\ ${ }^{1}$ Laboratory of Mathematics, Geometry, Analysis, Control and Applications, \\ Tahar Moulay University of Saïda, P.O. Box 138, EN-Nasr, 20000 Saïda, Algeria \\ ${ }^{2}$ Laboratory of Mathematics, Djillali Liabes University of Sidi Bel-Abbes, P.O. Box 89, Sidi Bel-Abbès 22000, Algeria \\ ${ }^{3}$ Departamento de Estatistica, Analisis Matematico e Optimizacion, Facultade de Matematicas, Universidade de Santiago de \\ Compostela, Santiago de Compostela, Spain
}

\begin{abstract}
In this paper, by using the technique that relies on the concept of the measure of noncompactness and the fixed point theory, we prove some existence results for some coupled systems of Hilfer and Hilfer-Hadamard fractional differential equations.
\end{abstract}

Keywords. Coupled system; Fixed point; Fractional differential equation; Hilfer-Hadamard fractional derivative; Measure of noncompactness.

2010 Mathematics Subject Classification. 26A33.

\section{INTRODUCTION}

Fractional differential equations have recently been applied in various areas of engineering, mathematics, physics and bio-engineering, and other applied sciences $[1,2]$. For some fundamental results in the theory of fractional calculus and fractional differential equations, we refer the reader to the monographs of Abbas, Benchohra and N'Guérékata [3, 4], Samko, Kilbas and Marichev [5], Kilbas, Srivastava and Trujillo [6] and Zhou [7], the papers by Abbas et al. [8, 9, 10], and the references therein. Recently, considerable attention have been given to the existence of solutions of initial and boundary value problems for fractional differential equations with Hilfer fractional derivative; see [1,11,12, 13, 14, 15, 16, 17] and the references therein and a coupled system of Hadamard type sequential fractional differential equations was considered in [18]. In [19, 20, 21], the measure of noncompactness was applied to some classes of functional Riemann-Liouville or Caputo fractional differential equations in Banach spaces. Motivated

\footnotetext{
${ }^{*}$ Corresponding author.

E-mail addresses: abbasmsaid@yahoo.fr (S. Abbas), benchohra@univ-sba.dz (M. Benchohra), lazregjamal@yahoo.fr (J.E. Lazreg), juanjose.nieto.roig@usc.es (J.J. Nieto).

Received September 27, 2017; Accepted March 4, 2018.
}

(C)2018 Journal of Nonlinear Functional Analysis 
by the research going on in this direction, we discuss the existence of solutions for the following coupled system of Hilfer fractional differential equations

$$
\left\{\begin{array}{l}
\left(D_{0}^{\alpha_{1}, \beta_{1}} u\right)(t)=f_{1}(t, u(t), v(t)) \\
\left(D_{0}^{\alpha_{2}, \beta_{2}} v\right)(t)=f_{2}(t, u(t), v(t))
\end{array} \quad ; t \in I:=[0, T],\right.
$$

with the following initial conditions

$$
\left\{\begin{array}{l}
\left(I_{0}^{1-\gamma_{1}} u\right)(0)=\phi_{1} \\
\left(I_{0}^{1-\gamma_{2}} v\right)(0)=\phi_{2}
\end{array}\right.
$$

where $T>0, \alpha_{i} \in(0,1), \beta_{i} \in[0,1], \gamma_{i}=\alpha_{i}+\beta_{i}-\alpha_{i} \beta_{i}, \phi_{i} \in E, f_{i}: I \times E \times E \rightarrow E ; i=1,2$, are given functions, $E$ is a real (or complex) Banach space with a norm $\|\cdot\|, I_{0}^{1-\gamma_{i}}$ is the left-sided mixed Riemann-Liouville integral of order $1-\gamma_{i}$, and $D_{0}^{\alpha_{i}, \beta_{i}}$ is the generalized Riemann-Liouville derivative (Hilfer) operator of order $\alpha_{i}$ and type $\beta_{i}: i=1,2$.

Next, we consider the following coupled system of Hilfer-Hadamard fractional differential equations

$$
\left\{\begin{array}{l}
\left({ }^{H} D_{1}^{\alpha_{1}, \beta_{1}} u\right)(t)=g_{1}(t, u(t), v(t)) \\
\left({ }^{H} D_{1}^{\alpha_{2}, \beta_{2}} v\right)(t)=g_{2}(t, u(t), v(t)),
\end{array} ; t \in[1, T],\right.
$$

with the following initial conditions

$$
\left\{\begin{array}{l}
\left({ }^{H} I_{1}^{1-\gamma_{1}} u\right)(1)=\psi_{1} \\
\left({ }^{H} I_{1}^{1-\gamma_{2}} v\right)(1)=\psi_{2}
\end{array}\right.
$$

where $T>1, \alpha_{i} \in(0,1), \beta_{i} \in[0,1], \gamma_{i}=\alpha_{i}+\beta_{i}-\alpha_{i} \beta_{i}, \psi_{i} \in E, g_{i}:[1, T] \times E \times E \rightarrow E ; i=1,2$ are given functions, ${ }^{H} I_{1}^{1-\gamma_{i}}$ is the left-sided mixed Hadamard integral of order $1-\gamma_{i}$, and ${ }^{H} D_{1}^{\alpha_{i}, \beta_{i}}$ is the Hilfer-Hadamard fractional derivative of order $\alpha_{i}$ and type $\beta_{i} ; i=1,2$.

\section{PRELIMINARIES}

Let $C$ be the Banach space of all continuous functions $v$ from $I$ into $E$ with the supremum (uniform) norm

$$
\|v\|_{\infty}:=\sup _{t \in I}\|v(t)\|
$$

As usual, by $A C(I)$ we denote the space of absolutely continuous functions from $I$ into $E$. We denote by $A C^{1}(I)$ the space defined by

$$
A C^{1}(I):=\left\{w: I \rightarrow E: \frac{d}{d t} w(t) \in A C(I)\right\} .
$$

By $L^{1}(I)$, we denote the space of measurable functions $v: I \rightarrow E$ which are Bochner integrable, with the norm

$$
\|v\|_{1}=\int_{0}^{T}|v(t)| d t
$$

By $C_{\gamma}(I)$ and $C_{\gamma}^{1}(I)$, we denote the weighted spaces of continuous functions defined by

$$
C_{\gamma}(I)=\left\{w:(0, T] \rightarrow E: t^{1-\gamma} w(t) \in C\right\},
$$

with the norm

$$
\|w\|_{C_{\gamma}}:=\sup _{t \in I}\left|t^{1-\gamma} w(t)\right|
$$


and

with the norm

$$
C_{\gamma}^{1}(I)=\left\{w \in C: \frac{d w}{d t} \in C_{\gamma}\right\}
$$

$$
\|w\|_{C_{\gamma}^{1}}:=\|w\|_{\infty}+\left\|w^{\prime}\right\|_{C_{\gamma}} .
$$

Also, by $\mathscr{C}:=C_{\gamma_{1}} \times C_{\gamma_{2}}$ we denote the product weighted space with the norm

$$
\|(u, v)\|_{\mathscr{C}}=\|u\|_{C_{\gamma_{1}}}+\|v\|_{C_{\gamma_{2}}}
$$

Let $\mathscr{M}_{X}$ denote the class of all bounded subsets of a metric space $X$.

Definition 2.1. Let $X$ be a complete metric space. A map $\mu: \mathscr{M}_{X} \rightarrow[0, \infty)$ is called a measure of noncompactness on $X$ if it satisfies the following properties for all $B, B_{1}, B_{2} \in \mathscr{M}_{X}$.

(a) $\mu(B)=0$ if and only if $B$ is precompact (Regularity),

(b) $\mu(B)=\mu(\bar{B})$ (Invariance under closure),

(c) $\mu\left(B_{1} \cup B_{2}\right)=\max \left\{\mu\left(B_{1}\right), \mu\left(B_{2}\right)\right\}$ (Semi-additivity).

Example 2.2. In every metric space $X$, the map $\phi: \mathscr{M}_{X} \rightarrow[0, \infty)$ with $\phi(B)=0$ if $B$ is relatively compact and $\phi(B)=1$ otherwise is a measure of noncompactness, the so-called discrete measure of noncompactness; see Example 1 in [19].

Definition 2.3. [20] Let $E$ be a Banach space and let $\Omega_{E}$ be the family of bounded subsets of $E$. The Kuratowski measure of noncompactness is the map $\mu: \Omega_{E} \rightarrow[0, \infty)$ defined by

$$
\mu(M)=\inf \left\{\varepsilon>0: M \subset \cup_{j=1}^{m} M_{j}, \operatorname{diam}\left(M_{j}\right) \leq \varepsilon\right\}
$$

where $M \in \Omega_{E}$.

Here, we list some important properties.

(1) $\mu(M)=0 \Leftrightarrow \bar{M}$ is compact ( $M$ is relatively compact).

(2) $\mu(M)=\mu(\bar{M})$.

(3) $M_{1} \subset M_{2} \Rightarrow \mu\left(M_{1}\right) \leq \mu\left(M_{2}\right)$.

(4) $\mu\left(M_{1}+M_{2}\right) \leq \mu\left(M_{1}\right)+\mu\left(M_{2}\right)$.

(5) $\mu(c M)=|c| \mu(M), c \in \mathbb{R}$.

(6) $\mu(\operatorname{conv} M)=\mu(M)$.

Now, we give some results and properties of fractional calculus.

Definition 2.4. $[3,5,6]$ The left-sided mixed Riemann-Liouville integral of order $r>0$ of a function $w \in L^{1}(I)$ is defined by

$$
\left(I_{0}^{r} w\right)(t)=\frac{1}{\Gamma(r)} \int_{0}^{t}(t-s)^{r-1} w(s) d s ; \text { for a.e. } t \in I,
$$

where $\Gamma(\cdot)$ is the (Euler's) Gamma function defined by

$$
\Gamma(\xi)=\int_{0}^{\infty} t^{\xi-1} e^{-t} d t ; \xi>0 .
$$

For all $r, r_{1}, r_{2}>0$ and each $w \in C$, we have $I_{0}^{r} w \in C$, and

$$
\left(I_{0}^{r_{1}} I_{0}^{r_{2}} w\right)(t)=\left(I_{0}^{r_{1}+r_{2}} w\right)(t) ; \text { for a.e. } t \in I .
$$


Definition 2.5. $[3,5,6]$ The Riemann-Liouville fractional derivative of order $r \in(0,1]$ of a function $w \in L^{1}(I)$ is defined by

$$
\begin{aligned}
\left(D_{0}^{r} w\right)(t) & =\left(\frac{d}{d t} I_{0}^{1-r} w\right)(t) \\
& =\frac{1}{\Gamma(1-r)} \frac{d}{d t} \int_{0}^{t}(t-s)^{-r} w(s) d s ; \text { for a.e. } t \in I .
\end{aligned}
$$

Let $r \in(0,1], \gamma \in[0,1)$ and $w \in C_{1-\gamma}(I)$. Then the following expression leads to the left inverse operator as follows

$$
\left(D_{0}^{r} I_{0}^{r} w\right)(t)=w(t) ; \text { for all } t \in(0, T] .
$$

Moreover, if $I_{0}^{1-r} w \in C_{1-\gamma}^{1}(I)$, then the following composition is proved in [5]

$$
\left(I_{0}^{r} D_{0}^{r} w\right)(t)=w(t)-\frac{\left(I_{0}^{1-r} w\right)\left(0^{+}\right)}{\Gamma(r)} t^{r-1} ; \text { for all } t \in(0, T] .
$$

Definition 2.6. $[3,6,5]$ The Caputo fractional derivative of order $r \in(0,1]$ of a function $w \in L^{1}(I)$ is defined by

$$
\begin{aligned}
\left({ }^{c} D_{0}^{r} w\right)(t) & =\left(I_{0}^{1-r} \frac{d}{d t} w\right)(t) \\
& =\frac{1}{\Gamma(1-r)} \int_{0}^{t}(t-s)^{-r} \frac{d}{d s} w(s) d s ; \text { for a.e. } t \in I .
\end{aligned}
$$

In [1], Hilfer studied applications of a generalized fractional operator having the Riemann-Liouville and the Caputo derivatives as specific cases (see also [14, 15].

Definition 2.7. (Hilfer derivative). Let $\alpha \in(0,1), \beta \in[0,1], w \in L^{1}(I)$, and

$$
I_{0}^{(1-\alpha)(1-\beta)} w \in A C^{1}(I) .
$$

The Hilfer fractional derivative of order $\alpha$ and type $\beta$ of $w$ is defined as

$$
\left(D_{0}^{\alpha, \beta} w\right)(t)=\left(I_{0}^{\beta(1-\alpha)} \frac{d}{d t} I_{0}^{(1-\alpha)(1-\beta)} w\right)(t) ; \text { for a.e. } t \in I .
$$

Here, we also list some properties.

Let $\alpha \in(0,1), \beta \in[0,1], \gamma=\alpha+\beta-\alpha \beta$, and $w \in L^{1}(I)$.

1. The operator $\left(D_{0}^{\alpha, \beta} w\right)(t)$ can be written as

$$
\left(D_{0}^{\alpha, \beta} w\right)(t)=\left(I_{0}^{\beta(1-\alpha)} \frac{d}{d t} I_{0}^{1-\gamma} w\right)(t)=\left(I_{0}^{\beta(1-\alpha)} D_{0}^{\gamma} w\right)(t) ; \text { for a.e. } t \in I .
$$

Moreover, the parameter $\gamma$ satisfies

$$
\gamma \in(0,1], \gamma \geq \alpha, \gamma>\beta, 1-\gamma<1-\beta(1-\alpha) .
$$

2. The generalization (2.1) for $\beta=0$ coincides with the Riemann-Liouville derivative and for $\beta=1$ with the Caputo derivative

$$
D_{0}^{\alpha, 0}=D_{0}^{\alpha}, \text { and } D_{0}^{\alpha, 1}={ }^{c} D_{0}^{\alpha} .
$$

3. If $D_{0}^{\beta(1-\alpha)} w$ exists and is in $L^{1}(I)$, then

$$
\left(D_{0}^{\alpha, \beta} I_{0}^{\alpha} w\right)(t)=\left(I_{0}^{\beta(1-\alpha)} D_{0}^{\beta(1-\alpha)} w\right)(t) ; \text { for a.e. } t \in I .
$$


Furthermore, if $w \in C_{\gamma}(I)$ and $I_{0}^{1-\beta(1-\alpha)} w \in C_{\gamma}^{1}(I)$, then

$$
\left(D_{0}^{\alpha, \beta} I_{0}^{\alpha} w\right)(t)=w(t) ; \text { for a.e. } t \in I .
$$

4. If $D_{0}^{\gamma} w$ exists and is in $L^{1}(I)$, then

$$
\left(I_{0}^{\alpha} D_{0}^{\alpha, \beta} w\right)(t)=\left(I_{0}^{\gamma} D_{0}^{\gamma} w\right)(t)=w(t)-\frac{I_{0}^{1-\gamma}\left(0^{+}\right)}{\Gamma(\gamma)} t^{\gamma-1} ; \text { for a.e. } t \in I .
$$

Corollary 2.8. Let $h \in C_{\gamma}(I)$. Then the Cauchy problem

$$
\left\{\begin{array}{l}
\left(D_{0}^{\alpha, \beta} u\right)(t)=h(t) ; t \in I \\
\left.\left(I_{0}^{1-\gamma} u\right)(t)\right|_{t=0}=\phi
\end{array}\right.
$$

has the following unique solution

$$
u(t)=\frac{\phi}{\Gamma(\gamma)} t^{\gamma-1}+\left(I_{0}^{\alpha} h\right)(t) .
$$

Lemma 2.9. [22] If $Y$ is a bounded subset of a Banach space $X$, then for each $\varepsilon>0$, there is a sequence $\left\{y_{k}\right\}_{k=1}^{\infty} \subset Y$ such that

$$
\mu(Y) \leq 2 \mu\left(\left\{y_{k}\right\}_{k=1}^{\infty}\right)+\varepsilon .
$$

Lemma 2.10. [23] If $\left\{u_{k}\right\}_{k=1}^{\infty} \subset L^{1}(I)$ is uniformly integrable, then $\mu\left(\left\{u_{k}\right\}_{k=1}^{\infty}\right)$ is measurable and for each $t \in I$,

$$
\mu\left(\left\{\int_{0}^{t} u_{k}(s) d s\right\}_{k=1}^{\infty}\right) \leq 2 \int_{0}^{t} \mu\left(\left\{u_{k}(s)\right\}_{k=1}^{\infty}\right) d s .
$$

In the sequel, we will make use of the following fixed point theorem.

Theorem 2.11. [24] Let $F$ be a closed and convex subset of a real Banach space. Let $G: F \rightarrow F$ be a continuous operator and Let $G(F)$ be bounded. If there exists a constant $k \in[0,1)$ such that for each bounded subset $B \subset F$,

$$
\mu(G(B)) \leq k \mu(B)
$$

then $G$ has a fixed point in $F$.

\section{A Coupled System of Hilfer Fractional Differential Equations}

In this section, we are concerned with the existence of solutions of system (1.1)-(1.2).

Definition 3.1. By a solution of problem (1.1)-(1.2), we mean a coupled measurable functions $(u, v) \in$ $C_{\gamma_{1}} \times C_{\gamma_{2}}$ those satisfy equations (1.1) on $I$, and conditions $\left(I_{0}^{1-\gamma_{1}} u\right)\left(0^{+}\right)=\phi_{1}$, and $\left(I_{0}^{1-\gamma_{2}} v\right)\left(0^{+}\right)=\phi_{2}$.

The following hypotheses will be used in the sequel.

$\left(H_{1}\right)$ The functions $t \mapsto f_{i}(t, u, v) ; i=1,2$ are measurable on $I$ for each $(u, v) \in C_{\gamma_{1}} \times C_{\gamma_{2}}$, and the functions $(u, v) \mapsto f_{i}(t, u, v)$ are continuous on $C_{\gamma_{1}} \times C_{\gamma_{2}}$ for a.e. $t \in I$,

$\left(H_{2}\right)$ There exist continuous functions $p_{i}: I \rightarrow(0, \infty) ; i=1,2$ such that

$$
\left\|f_{i}(t, u, v)-f_{i}(t, w, z)\right\| \leq \frac{p_{i}(t)\|u-w\|}{1+\|u-w\|+\|v-z\|} ; \text { for a.e. } t \in I, \text { and each } u, v, w, z \in E,
$$

$\left(H_{3}\right)$ For each bounded and measurable set $B \subset C_{\gamma_{i}} ; i=1,2$ and for each $t \in I$, we have

$$
\mu\left(f_{i}(t, B, B)\right) \leq p_{i}(t) \mu(B),
$$


Set

$$
p_{i}^{*}=\sup _{t \in I} p_{i}(t), \text { and } f_{i}^{*}=\sup _{t \in I}\left\|f_{i}(t, 0,0)\right\| ; i=1,2 .
$$

Now, we shall prove the following theorem concerning the existence of solutions of system (1.1)-(1.2).

Theorem 3.2. Assume that hypotheses $\left(H_{1}\right)-\left(H_{3}\right)$ hold. If

$$
\ell:=\frac{4 p_{1}^{*} T^{\alpha_{1}}}{\Gamma\left(1+\alpha_{1}\right)}+\frac{4 p_{2}^{*} T^{\alpha_{2}}}{\Gamma\left(1+\alpha_{2}\right)}<1
$$

then coupled system (1.1)-(1.2) has at least one solution defined on I.

Proof. Define the operators $N_{1}: C_{\gamma_{1}} \rightarrow C_{\gamma_{1}}$ and $N_{2}: C_{\gamma_{2}} \rightarrow C_{\gamma_{2}}$ by

$$
\left(N_{1} u\right)(t)=\frac{\phi_{1}}{\Gamma\left(\gamma_{1}\right)} t^{\gamma_{1}-1}+\int_{0}^{t}(t-s)^{\alpha_{1}-1} \frac{f(s, u(s), v(s))}{\Gamma\left(\alpha_{1}\right)} d s,
$$

and

$$
\left(N_{2} v\right)(t)=\frac{\phi_{2}}{\Gamma\left(\gamma_{2}\right)} t^{\gamma_{2}-1}+\int_{0}^{t}(t-s)^{\alpha_{2}-1} \frac{f(s, u(s), v(s))}{\Gamma\left(\alpha_{2}\right)} d s
$$

Consider the continuous operator $N: \mathscr{C} \rightarrow \mathscr{C}$ defined by

$$
(N(u, v))(t)=\left(\left(N_{1} u\right)(t),\left(N_{2} v\right)(t)\right) .
$$

Clearly, the fixed points of the operator $N$ are solutions of system (1.1)-(1.2). For any $u \in C_{\gamma_{1}}$, and each $t \in I$, we have

$$
\begin{aligned}
\left\|t^{1-\gamma_{1}}\left(N_{1} u\right)(t)\right\| \leq & \frac{\left\|\phi_{1}\right\|}{\Gamma\left(\gamma_{1}\right)}+\frac{t^{1-\gamma_{1}}}{\Gamma\left(\alpha_{1}\right)} \int_{0}^{t}(t-s)^{\alpha_{1}-1}\left\|f_{1}(s, u(s), v(s))\right\| d s \\
\leq & \frac{\left\|\phi_{1}\right\|}{\Gamma\left(\gamma_{1}\right)}+\frac{t^{1-\gamma_{1}}}{\Gamma\left(\alpha_{1}\right)} \int_{0}^{t}(t-s)^{\alpha_{1}-1}\left\|f_{1}(s, 0,0)\right\| d s \\
& +\frac{t^{1-\gamma_{1}}}{\Gamma\left(\alpha_{1}\right)} \int_{0}^{t}(t-s)^{\alpha_{1}-1}\left\|f_{1}(s, u(s), v(s))-f_{1}(s, 0,0)\right\| d s \\
\leq & \frac{\left\|\phi_{1}\right\|}{\Gamma\left(\gamma_{1}\right)}+\frac{t^{1-\gamma_{1}}}{\Gamma\left(\alpha_{1}\right)} \int_{0}^{t}(t-s)^{\alpha_{1}-1}\left(\left\|f_{1}(s, 0,0)\right\|+p_{1}(s)\right) d s \\
\leq & \frac{\left\|\phi_{1}\right\|}{\Gamma\left(\gamma_{1}\right)}+\frac{\left(f_{1}^{*}+p_{1}^{*}\right) T^{1-\gamma_{1}}}{\Gamma\left(\alpha_{1}\right)} \int_{0}^{t}(t-s)^{\alpha_{1}-1} d s \\
\leq & \frac{\left\|\phi_{1}\right\|}{\Gamma\left(\gamma_{1}\right)}+\frac{\left(f_{1}^{*}+p_{1}^{*}\right) T^{1-\gamma_{1}+\alpha_{1}}}{\Gamma\left(1+\alpha_{1}\right)} .
\end{aligned}
$$

Also, for any $v \in C_{\gamma_{2}}$, and each $t \in I$, we get

$$
\left\|t^{1-\gamma_{1}}\left(N_{1} u\right)(t)\right\| \leq \frac{\left\|\phi_{2}\right\|}{\Gamma\left(\gamma_{2}\right)}+\frac{\left(f_{2}^{*}+p_{2}^{*}\right) T^{1-\gamma_{2}+\alpha_{2}}}{\Gamma\left(1+\alpha_{2}\right)} .
$$

Thus

$$
\|N(u, v)\|_{\mathscr{C}} \leq \frac{\left\|\phi_{1}\right\|}{\Gamma\left(\gamma_{1}\right)}+\frac{\left\|\phi_{2}\right\|}{\Gamma\left(\gamma_{2}\right)}+\frac{\left(f_{1}^{*}+p_{1}^{*}\right) T^{1-\gamma_{1}+\alpha_{1}}}{\Gamma\left(1+\alpha_{1}\right)}+\frac{\left(f_{2}^{*}+p_{2}^{*}\right) T^{1-\gamma_{2}+\alpha_{2}}}{\Gamma\left(1+\alpha_{2}\right)}:=R .
$$

This proves that $N$ transforms the ball

$$
B_{R}:=B(0, R)=\left\{(u, v) \in \mathscr{C}:\|(u, v)\|_{\mathscr{C}} \leq R\right\}
$$

into itself. We next show that the operator $N: B_{R} \rightarrow B_{R}$ satisfies all the assumptions of Theorem 2.11. The proof will be given in several steps. 
Step 1. $N: B_{R} \rightarrow B_{R}$ is continuous.

Let $\left\{\left(u_{n}, v_{n}\right)\right\}_{n \in \mathbb{N}}$ be a sequence such that $\left(u_{n}, v_{n}\right) \rightarrow(u, v)$ in $B_{R}$. Then, for each $t \in I$, we have

$$
\begin{aligned}
& \left\|t^{1-\gamma_{1}}\left(N_{1} u_{n}\right)(t)-t^{1-\gamma_{1}}\left(N_{1} u\right)(t)\right\| \\
& \leq \frac{t^{1-\gamma_{1}}}{\Gamma\left(\alpha_{1}\right)} \int_{0}^{t}(t-s)^{\alpha_{1}-1}\left\|f_{1}\left(s, u_{n}(s), v_{n}(s)\right)-f_{1}(s, u(s), v(s))\right\| d s \\
& \leq \frac{p_{1}^{*} T^{1-\gamma_{1}}}{\Gamma\left(\alpha_{1}\right)} \int_{0}^{t}(t-s)^{\alpha_{1}-1}\left\|u_{n}(s)-u(s)\right\| d s .
\end{aligned}
$$

Also, $\left\|t^{1-\gamma_{2}}\left(N_{2} v_{n}\right)(t)-t^{1-\gamma_{2}}\left(N_{2} v\right)(t)\right\| \leq \frac{p_{2}^{*} T^{1-\gamma_{2}}}{\Gamma\left(\alpha_{2}\right)} \int_{0}^{t}(t-s)^{\alpha_{2}-1}\left\|u_{n}(s)-u(s)\right\| d s$. Hence

$$
\begin{aligned}
& \left\|t^{1-\gamma_{1}}\left(N_{1} u_{n}\right)(t)-t^{1-\gamma_{1}}\left(N_{1} u\right)(t)\right\|+\left\|t^{1-\gamma_{2}}\left(N_{2} v_{n}\right)(t)-t^{1-\gamma_{2}}\left(N_{2} v\right)(t)\right\| \\
& \leq \frac{p_{1}^{*} T^{1-\gamma_{1}}}{\Gamma\left(\alpha_{1}\right)} \int_{0}^{t}(t-s)^{\alpha_{1}-1}\left\|u_{n}(s)-u(s)\right\| d s+\frac{p_{2}^{*} T^{1-\gamma_{2}}}{\Gamma\left(\alpha_{2}\right)} \int_{0}^{t}(t-s)^{\alpha_{2}-1}\left\|u_{n}(s)-u(s)\right\| d s .
\end{aligned}
$$

Since $u_{n} \rightarrow u$ as $n \rightarrow \infty$, we see that equation (3.6) implies $\left\|N\left(u_{n}, v_{n}\right)-N(u, v)\right\|_{\mathscr{C}} \rightarrow 0$ as $n \rightarrow \infty$.

Step 2. $N\left(B_{R}\right)$ is bounded.

Since $N\left(B_{R}\right) \subset B_{R}$ and $B_{R}$ is bounded, we find that $N\left(B_{R}\right)$ is bounded.

Step 3. For each bounded subset $D$ of $B_{R}, \mu(N(D)) \leq \ell \mu(D)$.

From Lemmas 2.9 and 2.10, for any $D \subset B_{R}$ and any $\varepsilon>0$, there exists a sequence $\left\{\left(u_{n}, v_{n}\right)\right\}_{n=0}^{\infty} \subset D$, such that, for all $t \in I$,

$$
\begin{aligned}
\mu((N D)(t))= & \mu\left(\left\{\frac{\phi_{1}}{\Gamma\left(\gamma_{1}\right)} t^{\gamma_{1}-1}+\int_{0}^{t}(t-s)^{\alpha_{1}-1} \frac{f_{1}(s, u(s), v(s))}{\Gamma\left(\alpha_{1}\right)} d s\right.\right. \\
& \left.\left.+\frac{\phi_{2}}{\Gamma\left(\gamma_{2}\right)} t^{\gamma_{2}-1}+\int_{0}^{t}(t-s)^{\alpha_{2}-1} \frac{f_{2}(s, u(s), v(s))}{\Gamma\left(\alpha_{2}\right)} d s ;(u, v) \in D\right\}\right) \\
\leq & 2 \mu\left(\left\{\int_{0}^{t} \frac{(t-s)^{\alpha_{1}-1}}{\Gamma\left(\alpha_{1}\right)} f_{1}\left(s, u_{n}(s), v_{n}(s)\right) d s\right.\right. \\
& \left.\left.+\int_{0}^{t} \frac{(t-s)^{\alpha_{2}-1}}{\Gamma\left(\alpha_{2}\right)} f_{2}\left(s, u_{n}(s), v_{n}(s)\right) d s\right\}_{n=1}^{\infty}\right)+\varepsilon \\
\leq & 4 \int_{0}^{t} \frac{(t-s)^{\alpha_{1}-1}}{\Gamma\left(\alpha_{1}\right)} \mu\left(\left\{f_{1}\left(s, u_{n}(s), v_{n}(s)\right)\right\}_{n=1}^{\infty}\right) d s \\
& +4 \int_{0}^{t} \frac{(t-s)^{\alpha_{2}-1}}{\Gamma\left(\alpha_{2}\right)} \mu\left(\left\{f_{2}\left(s, u_{n}(s), v_{n}(s)\right)\right\}_{n=1}^{\infty}\right) d s+\varepsilon \\
\leq & 4\left(\int_{0}^{t} \frac{(t-s)^{\alpha_{1}-1}}{\Gamma\left(\alpha_{1}\right)} p_{1}(s)+\int_{0}^{t} \frac{(t-s)^{\alpha_{2}-1}}{\Gamma\left(\alpha_{2}\right)} p_{2}(s)\right) \mu\left(\left\{\left(u_{n}(s), v_{n}(s)\right)\right\}_{n=1}^{\infty}\right) d s+\varepsilon \\
\leq & 4\left(\int_{0}^{t} \frac{(t-s)^{\alpha_{1}-1}}{\Gamma\left(\alpha_{1}\right)} p_{1}(s) d s+\int_{0}^{t} \frac{(t-s)^{\alpha_{2}-1}}{\Gamma\left(\alpha_{2}\right)} p_{2}(s) d s\right) \mu\left(\left\{\left(u_{n}, v_{n}\right)\right\}_{n=1}^{\infty}\right)+\varepsilon \\
\leq & 4\left(\int_{0}^{t} \frac{(t-s)^{\alpha_{1}-1}}{\Gamma\left(\alpha_{1}\right)} p_{1}(s) d s+\int_{0}^{t} \frac{(t-s)^{\alpha_{2}-1}}{\Gamma\left(\alpha_{2}\right)} p_{2}(s) d s\right) \mu(D)+\varepsilon \\
\leq & 4\left(\frac{p_{1}^{*} T^{\alpha_{1}}}{\Gamma\left(1+\alpha_{1}\right)}+\frac{p_{2}^{*} T^{\alpha_{2}}}{\Gamma\left(1+\alpha_{2}\right)}\right) \mu(D)+\varepsilon \\
\leq & \ell \mu(D)+\varepsilon .
\end{aligned}
$$


Since $\varepsilon>0$ is arbitrary, we ahve

$$
\mu(N(B)) \leq \ell \mu(B) .
$$

As a consequence of Steps 1 to 3 together with Theorem 2.11, we can conclude that $N$ has at least one fixed point in $B_{R}$ which is a solution of system (1.1)- (1.2).

\section{A Coupled System of Hilfer-Hadamard Fractional Differential Equations}

Now, we are concerned with the existence of solutions of coupled system (1.3)-(1.4).

Set $C:=C([1, T])$, and denote the weighted space of continuous functions defined by

$$
C_{\gamma, \ln }([1, T])=\left\{w(t):(\ln t)^{1-\gamma} w(t) \in C\right\},
$$

with the norm

$$
\|w\|_{C_{\gamma, \mathrm{In}}}:=\sup _{t \in[1, T]}\left|(\ln t)^{1-r} w(t)\right| .
$$

Also, by $\mathscr{C}_{\gamma_{1}, \gamma_{2}, \ln }([1, T]):=C_{\gamma_{1}, \ln }([1, T]) \times C_{\gamma_{2}, \ln }([1, T])$ we denote the product weighted space with the norm

$$
\|(u, v)\|_{\mathscr{C}_{\gamma_{1}, \gamma_{2}, \ln }([1, T])}=\|u\|_{C_{\gamma_{1}, \ln }}+\|v\|_{C_{\gamma_{2}, \ln }} .
$$

Let us recall some definitions and properties of Hadamard fractional integration and differentiation. We refer to [6] for a more detailed analysis.

Definition 4.1. [6] (Hadamard fractional integral). The Hadamard fractional integral of order $q>0$ for a function $g \in L^{1}([1, T])$, is defined as

$$
\left({ }^{H} I_{1}^{q} g\right)(x)=\frac{1}{\Gamma(q)} \int_{1}^{x}\left(\ln \frac{x}{s}\right)^{q-1} \frac{g(s)}{s} d s,
$$

provided the integral exists.

Example 4.2. Let $0<q<1$ and $g(x)=\ln x, x \in[0, e]$. Then

$$
\left({ }^{H} I_{1}^{q} g\right)(x)=\frac{1}{\Gamma(2+q)}(\ln x)^{1+q} ; \text { for a.e. } x \in[0, e] .
$$

Set

$$
\delta=x \frac{d}{d x}, q>0, n=[q]+1
$$

and

$$
A C_{\delta}^{n}:=\left\{u:[1, T] \rightarrow E: \delta^{n-1}[u(x)] \in A C(I)\right\} .
$$

Analogous to the Riemann-Liouville fractional derivative, the Hadamard fractional derivative is defined in terms of the Hadamard fractional integral in the following way:

Definition 4.3. [6] (Hadamard fractional derivative). The Hadamard fractional derivative of order $q>0$ applied to the function $w \in A C_{\delta}^{n}$ is defined as

$$
\left({ }^{H} D_{1}^{q} w\right)(x)=\delta^{n}\left({ }^{H} I_{1}^{n-q} w\right)(x) .
$$

In particular, if $q \in(0,1]$, then

$$
\left({ }^{H} D_{1}^{q} w\right)(x)=\delta\left({ }^{H} I_{1}^{1-q} w\right)(x) .
$$


Example 4.4. Let $0<q<1$. Let $w(x)=\ln x, x \in[0, e]$. Then

$$
\left({ }^{H} D_{1}^{q} w\right)(x)=\frac{1}{\Gamma(2-q)}(\ln x)^{1-q} ; \text { for a.e. } x \in[0, e] .
$$

It has been proved (see, e.g., Kilbas [[25], Theorem 4.8]) that in the space $L^{1}(I)$, the Hadamard fractional derivative is the left-inverse operator to the Hadamard fractional integral, i.e.,

$$
\left({ }^{H} D_{1}^{q}\right)\left({ }^{H} I_{1}^{q} w\right)(x)=w(x) .
$$

From Theorem 2.3 of [6], we have

$$
\left({ }^{H} I_{1}^{q}\right)\left({ }^{H} D_{1}^{q} w\right)(x)=w(x)-\frac{\left({ }^{H} I_{1}^{1-q} w\right)(1)}{\Gamma(q)}(\ln x)^{q-1} .
$$

Analogous to the Hadamard fractional calculus, the Caputo-Hadamard fractional derivative is defined in the following way.

Definition 4.5. (Caputo-Hadamard fractional derivative) The Caputo-Hadamard fractional derivative of order $q>0$ applied to the function $w \in A C_{\delta}^{n}$ is defined as

$$
\left({ }^{H c} D_{1}^{q} w\right)(x)=\left({ }^{H} I_{1}^{n-q} \delta^{n} w\right)(x) .
$$

In particular, if $q \in(0,1]$, then

$$
\left({ }^{H c} D_{1}^{q} w\right)(x)=\left({ }^{H} I_{1}^{1-q} \delta w\right)(x) .
$$

From the Hadamard fractional integral, the Hilfer-Hadamard fractional derivative (introduced for the first time in [26]) is defined in the following way.

Definition 4.6. (Hilfer-Hadamard fractional derivative). Let $\alpha \in(0,1), \beta \in[0,1], \gamma=\alpha+\beta-\alpha \beta, w \in$ $L^{1}(I)$, and ${ }^{H} I_{1}^{(1-\alpha)(1-\beta)} w \in A C^{1}(I)$. The Hilfer-Hadamard fractional derivative of order $\alpha$ and type $\beta$ applied to the function $w$ is defined as

$$
\begin{aligned}
\left({ }^{H} D_{1}^{\alpha, \beta} w\right)(t) & =\left({ }^{H} I_{1}^{\beta(1-\alpha)}\left({ }^{H} D_{1}^{\gamma} w\right)\right)(t) \\
& =\left({ }^{H} I_{1}^{\beta(1-\alpha)} \delta\left({ }^{H} I_{1}^{1-\gamma} w\right)\right)(t) ; \text { for a.e. } t \in[1, T] .
\end{aligned}
$$

This new fractional derivative (4.1) may be viewed as interpolating the Hadamard fractional derivative and the Caputo-Hadamard fractional derivative. Indeed for $\beta=0$ this derivative reduces to the Hadamard fractional derivative and when $\beta=1$, we recover the Caputo-Hadamard fractional derivative.

$$
{ }^{H} D_{1}^{\alpha, 0}={ }^{H} D_{1}^{\alpha}, \text { and }{ }^{H} D_{1}^{\alpha, 1}={ }^{H c} D_{1}^{\alpha} .
$$

From Theorem 21 of [27], we concluded the following lemma.

Lemma 4.7. Let $g:[1, T] \times E \rightarrow E$ be such that $g(\cdot, u(\cdot)) \in C_{\gamma, \ln }([1, T])$ for any $u \in C_{\gamma, \ln }([1, T])$. Then problem (1.3) is equivalent to the following Volterra integral equation

$$
u(t)=\frac{\phi_{0}}{\Gamma(\gamma)}(\ln t)^{\gamma-1}+\left({ }^{H} I_{1}^{\alpha} g(\cdot, u(\cdot))\right)(t) .
$$

Definition 4.8. By a solution of coupled system (1.3)-(1.4), we mean a coupled measurable functions $(u, v) \in C_{\gamma_{1}, \ln } \times C_{\gamma_{2}, \ln }$ those satisfy the conditions (1.4) and the equations (1.3) on $[1, T]$. 
Now we give (without proof) similar existence results for system (1.3)-(1.4). Let us introduce the following hypotheses:

$\left(H_{1}^{\prime}\right)$ The functions $t \mapsto g_{i}(t, u, v) ; i=1,2$ are measurable on $[1, T]$ for each $(u, v) \in C_{\gamma_{1}, \ln } \times C_{\gamma_{2}, \ln }$, and the function $(u, v) \mapsto g_{i}(t, u, v)$ is continuous on $C_{\gamma_{1}, \ln } \times C_{\gamma_{2}, \ln }$ for a.e. $t \in[1, T]$,

$\left(H_{2}^{\prime}\right)$ There exist continuous functions $q_{i}:[1, T] \rightarrow(0, \infty)$ such that

$$
\left\|g_{i}(t, u, v)-g_{i}(t, w, z)\right\| \leq \frac{q_{i}(t)\|u-w\|}{1+\|u-w\|+\|v-z\|} ; \text { for a.e. } t \in[1, T] \text {, and each } u, v, w, z \in E,
$$

$\left(H_{3}^{\prime}\right)$ For each bounded and measurable set $B \subset C_{\gamma_{1}, \gamma_{2}}$,n and for each $t \in[1, T]$, we have

$$
\mu\left(g_{i}(t, B, B)\right) \leq q_{i}(t) \mu(B) ; i=1,2 .
$$

Theorem 4.9. Assume that hypotheses $\left(H_{1}^{\prime}\right)-\left(H_{3}^{\prime}\right)$ hold. If

$$
\ell^{*}:=\frac{4 q_{1}^{*}(\ln T)^{\alpha_{1}}}{\Gamma\left(1+\alpha_{1}\right)}+\frac{4 q_{2}^{*}(\ln T)^{\alpha_{2}}}{\Gamma\left(1+\alpha_{2}\right)}<1
$$

where $q_{i}^{*}=\sup _{t \in I} q_{i}(t) ; i=1,2$, then system (1.3) -(1.4) has at least one solution defined on $[1, T]$.

\section{AN EXAMPLE}

Let

$$
E=l^{1}=\left\{u=\left(u_{1}, u_{2}, \ldots, u_{n}, \ldots\right), \sum_{n=1}^{\infty}\left|u_{n}\right|<\infty\right\}
$$

be the Banach space with the norm

$$
\|u\|_{E}=\sum_{n=1}^{\infty}\left|u_{n}\right|
$$

Consider the following coupled system of Hilfer fractional differential equation of the form

$$
\left\{\begin{array}{l}
\left(D_{0}^{\frac{1}{2}, \frac{1}{2}} u_{n}\right)(t)=f_{n}(t, u(t), v(t)) \\
\left(D_{0}^{\frac{1}{2}, \frac{1}{2}} v_{n}\right)(t)=g_{n}(t, u(t), v(t)) ; \\
\left.\left(I_{0}^{\frac{1}{4}} u_{n}\right)(t)\right|_{t=0}=(1,0, \ldots, 0, \ldots), \quad ; t \in[0,1] \\
\left.\left(I_{0}^{\frac{1}{4}} v_{n}\right)(t)\right|_{t=0}=(1,0, \ldots, 0, \ldots)
\end{array}\right.
$$

where

$$
\begin{aligned}
& \left\{\begin{array}{c}
f_{n}(t, u, v)=\frac{t^{\frac{-1}{4}} u_{n}(t) \sin t}{64(1+\sqrt{t})\left(1+\|u\|_{E}+\|v\|_{E}\right)} ; t \in(0,1], \\
f_{n}(0, u, v)=0
\end{array}\right. \\
& g_{n}(t, u, v)=\frac{u_{n}(t) \cos t}{64\left(1+\|u\|_{E}+\|v\|_{E}\right)} ; t \in(0,1]
\end{aligned}
$$

with

$$
\begin{gathered}
f=\left(f_{1}, f_{2}, \ldots, f_{n}, \ldots\right), g=\left(g_{1}, g_{2}, \ldots, g_{n}, \ldots\right), u=\left(u_{1}, u_{2}, \ldots, u_{n}, \ldots\right), \\
v=\left(v_{1}, v_{2}, \ldots, v_{n}, \ldots\right), \text { and } c:=\frac{e^{3}}{8} \Gamma\left(\frac{1}{2}\right) .
\end{gathered}
$$


Setting $\alpha_{i}=\beta_{i}=\frac{1}{2} ; i=1,2$, one has $\gamma_{i}=\frac{3}{4} ; i=1,2$. The hypothesis $\left(H_{2}\right)$ is satisfied with

$$
\left\{\begin{array}{l}
p_{1}(t)=\frac{t^{\frac{-1}{4}}|\sin t|}{64(1+\sqrt{t})} ; t \in(0,1], \\
p_{1}(0)=0
\end{array}\right.
$$

and

$$
p_{2}(t)=\frac{|\cos t|}{64} ; t \in(0,1] .
$$

Hence, Theorem 3.2 implies that system (5.1) has at least one solution defined on $[0,1]$.

\section{REFERENCES}

[1] R. Hilfer, Applications of Fractional Calculus in Physics, World Scientific, Singapore, 2000.

[2] V. E. Tarasov, Fractional Dynamics: Application of Fractional Calculus to Dynamics of Particles, Fields and Media, Springer, 2010.

[3] S. Abbas, M. Benchohra, G. M. N'Guérékata, Topics in Fractional Differential Equations, Springer, New York, 2012.

[4] S. Abbas, M. Benchohra, G. M. N'Guérékata, Advanced Fractional Differential and Integral Equations, Nova Science Publishers, New York, 2015.

[5] S. G. Samko, A. A. Kilbas, O. I. Marichev, Fractional Integrals and Derivatives, Gordon and Breach Science Publishers, Yverdon, 1993.

[6] A. A. Kilbas, H. M. Srivastava, J. J. Trujillo, Theory and Applications of Fractional Differential Equations, Elsevier Science B.V., Amsterdam, 2006.

[7] Y. Zhou, Basic Theory of Fractional Differential Equations, World Scientific, Singapore, 2014.

[8] S. Abbas, E. Alaidarous, M. Benchohra, J.J. Nieto, Existence and stability of solutions for Hadamard-Stieltjes fractional integral equations, Discret. Dyn. Nat. Soc. 2015 (2015), Article ID 317094.

[9] S. Abbas, M. Benchohra, J. Henderson, J.E. Lazreg, Measure of noncompactness and impulsive Hadamard fractional implicit differential equations in Banach spaces, Math. Eng. Science Aerospace 8 (2017), 1-19.

[10] S. Abbas, M. Benchohra, J.E. Lazreg, Y. Zhou, A Survey on Hadamard and Hilfer fractional differential equations: analysis and stability, Chaos, Solitons Fractals 102 (2017), 47-71.

[11] K. M. Furati, M. D. Kassim. Non-existence of global solutions for a differential equation involving Hilfer fractional derivative, Electron. J. Differential Equations 2013 (2013), Article ID 235.

[12] K. M. Furati, M. D. Kassim, and N. e-. Tatar, Existence and uniqueness for a problem involving Hilfer fractional derivative, Comput. Math. Appl. 64 (2012), 1616-1626.

[13] H. Gu, J.J. Trujillo, Existence of mild solution for evolution equation with Hilfer fractional derivative, Appl. Math. Comput. 257 (2015), 344-354.

[14] R. Kamocki, C. Obczńnski, On fractional Cauchy-type problems containing Hilfer's derivative, Electron. J. Qual. Theory Differ. Equ. 2016 (2016), Article ID 50.

[15] Ž. Tomovski, R. Hilfer, H. M. Srivastava, Fractional and operational calculus with generalized fractional derivative operators and Mittag-Leffler type functions, Integral Transforms Spec. Funct. 21 (2010), 797-814.

[16] J.-R. Wang, Y. Zhang, Nonlocal initial value problems for differential equations with Hilfer fractional derivative, Appl. Math. Comput. 266 (2015), 850-859.

[17] M. Yang, Q.-R. Wang, Approximate controllability of Hilfer fractional differential inclusions with nonlocal conditions, Math. Methods Appl. Sci. 40 (2017), 1126-1138.

[18] S. Aljoudi, B. Ahmad, J. Nieto, A. Alsaedi, A coupled system of Hadamard type sequential fractional differential equations with coupled strip conditions, Chaos, Solitons Fractals 91 (2016), 39-46.

[19] J. M. Ayerbee Toledano, T. Dominguez Benavides, G. López Acedo, Measures of Noncompactness in Metric Fixed Point Theory, Operator Theory, Advances and Applications, vol 99, Birkhäuser, Basel, Boston, Berlin, 1997.

[20] J. Banas̀, K. Goebel, Measures of Noncompactness in Banach Spaces, Marcel Dekker, New York, 1980.

[21] M. Benchohra, J. Henderson, D. Seba, Measure of noncompactness and fractional differential equations in Banach spaces, Commun. Appl. Anal. 12 (2008), 419-428. 
[22] D. Bothe, Multivalued perturbation of m-accretive differential inclusions, Isreal J. Math. 108 (1998), 109-138.

[23] H. Mönch, Boundary value problems for nonlinear ordinary differential equations of second order in Banach spaces, Nonlinear Anal. 4 (1980), 985-999.

[24] L. Liu, F. Guo, C. Wu, Y. Wu, Existence theorems of global solutions for nonlinear Volterra type integral equations in Banach spaces, J. Math. Anal. Appl. 309 (2005), 638-649.

[25] A. A. Kilbas, Hadamard-type fractional calculus, J. Korean Math. Soc. 38 (2001), 1191-1204.

[26] M. D. Qassim, K. M. Furati, N.-e. Tatar, On a differential equation involving Hilfer-Hadamard fractional derivative, Abst. Appl. Anal. 2012 (2012), Article ID 391062.

[27] M. D. Qassim, N.-e. Tatar, Well-posedness and stability for a differential problem with Hilfer-Hadamard fractional derivative, Abst. Appl. Anal. 2013 (2013), Article ID 605029. 